\title{
Corrigendum: On dynamics and bifurcations of area-preserving maps with homoclinic tangencies (2015 Nonlinearity 283027 )
}

\author{
Amadeu Delshams ${ }^{1}$, Marina Gonchenko ${ }^{2}$ \\ and Sergey Gonchenko ${ }^{3}$ \\ 1 Departament de Matemàtica Aplicada I, Universitat Politècnica de Catalunya, Di- \\ agonal 647, 08028 Barcelona, Spain \\ ${ }^{2}$ Universitat de Barcelona, Barcelona, Spain \\ ${ }^{3}$ Institute of Applied Mathematics and Cybernetics, Nizhny Novgorod State Univer- \\ sity Ulyanova St 10, 603005 Nizhny Novgorod, Russia \\ E-mail: Amadeu.Delshams@upc.edu \\ Received 20 January 2017 \\ Accepted for publication 20 January 2017 \\ Published 8 February 2017

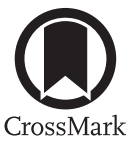

The authors wish to inform readers that a section of the results of the paper related to the case of area-preserving maps on non-orientable surfaces was published in our previous paper [1]. Unfortunately, the reference to [1] was missing. The authors wish to apologize for this oversight.

[1] Delshams A, Gonchenko M and Gonchenko S V 2014 On bifurcations of area-preserving and nonorientable maps with quadratic homoclinic tangencies Regular Chaotic Dyn. 19 702-17 